\title{
Prepubertal children born large for gestational age have lower serum DHEAS concentrations than those with a lower birth weight
}

\author{
Henrikki Nordman ${ }^{1}$, Raimo Voutilainen ${ }^{1}$, Leena Antikainen ${ }^{1}$ and Jarmo Jääskeläinen ${ }^{1}$
}

BACKGROUND: Children born small for gestational age (SGA) have higher serum dehydroepiandrosterone sulfate (DHEAS) concentrations than children born appropriate for gestational age (AGA). The overall metabolic risk associated with birth weight is U-shaped, but it is not known whether children born large for gestational age (LGA) have elevated serum DHEAS levels.

METHODS: A cohort of 49 children born LGA, 56 children born AGA, and 23 children born SGA were studied at 5-8 years of age. Anthropometric data at birth, at the age of 2 years, and at examination were recorded. Fasting blood samples were collected for serum analyses of DHEAS, insulinlike growth factor 1 (IGF-1), and insulin concentrations.

RESULTS: The children born LGA had lower serum DHEAS levels adjusted for body mass index (BMI) standard deviation score (SDS) and age than the rest of the children. Lower birth weight SDS and higher weight gain during the first 2 years of life predicted higher serum DHEAS levels. Higher serum IGF-1 levels were also associated with higher prevalence of adrenarchal DHEAS levels.

CONCLUSION: Being born LGA was associated with lower DHEAS levels, whereas small birth size and early catch-up growth predicted higher levels. This suggests that genetic or early epigenetic factors have an impact on adrenarche. IGF-1 may be a mediator in this process.

C hildren born small for gestational age (SGA) have higher serum dehydroepiandrosterone sulfate (DHEAS) concentrations than children born appropriate for gestational age (AGA) $(1,2)$. High prepubertal DHEAS levels are related to premature adrenarche (PA), defined as androgenic clinical signs appearing before the age of 8 years in girls and 9 years in boys in the presence of elevated serum DHEAS levels for age (reviewed in (refs 3-5)). PA has been associated with several metabolic disturbances including hyperinsulinemia/decreased insulin sensitivity $(6,7)$, adverse lipid profile (8), and increased fat mass and body fat percentage (9).

It is also well known that both children born SGA and those born large for gestational age (LGA) have an increased cardiometabolic disease risk $(10,11)$. They may develop obesity, type 2 diabetes, the metabolic syndrome, and hypertension later in life (11-16). However, it has not been studied whether LGA children have elevated serum DHEAS levels in childhood and/or an increased prevalence of PA.

PA has been associated with increased body weight $(9,17)$, and girls with PA have accelerated statural growth already during the first 2 years of life $(18,19)$. Early rapid weight gain of children with normal birth weight predicts higher DHEAS levels at the age of 8 years (20).

The purpose of this study was to investigate the influence of large birth size on serum DHEAS levels and the prevalence of adrenarche in prepubertal children.

\section{METHODS}

A cohort of 128 Caucasian children (67 boys) born singleton at term between 2004 and 2007 in Eastern Finland was studied (21). In brief, the children were enrolled according to their birth size and studied at 5.0-8.7 years of age (mean (95\% CI), 6.9 (6.8-7.1) years) (Table 1). SGA was defined as gender-specific birth weight $\leqslant-2.0$ SDS, LGA as birth weight $\geqslant+2.0$ SDS, and AGA as birth weight and length being between -1.0 and +1.0 SDS. Anthropometric data at birth, at the age of 2 years, and at examination were recorded (Table 1).

BMI was calculated as the body weight divided by the square of the height $\left(\mathrm{kg} / \mathrm{m}^{2}\right)$. Sex- and age-specific SDS for height and BMI were calculated according to the recently published Finnish growth reference (22). The following BMI SDS cutoff points for overweight and obesity were used corresponding to BMI of 25 and 30 at the age of 18 years: for boys, 0.78 and 1.70, and for girls, 1.16 and 2.11, respectively (22). Catch-up or catch-down growth was defined as an increase or a decrease in weight SDS $>0.67$ during the first 2 years of life, respectively (23).

Clinical signs of adrenarche (adult-type body odor, oily hair, acne or comedones, and appearance of pubic and axillary hair) and pubertal status (breast development in girls, testicular size in boys, and pubic hair in both sexes) were recorded. Children were defined prepubertal when girls had no palpable breast tissue or boys had a testicular volume $\leq 3 \mathrm{ml}$.

Fasting blood samples were collected for serum analyses of DHEAS, IGF-1, and insulin concentrations. DHEAS level of $1.0 \mu \mathrm{mol} / \mathrm{l}(37 \mu \mathrm{g} / \mathrm{dl})$ was referred as a cutoff for biochemical adrenarche $(24,25)$. Serum DHEAS and insulin concentrations were analyzed using electrochemiluminescence immunoassay (Roche Diagnostics GmbH, Mannheim, Germany). Serum IGF-1

\footnotetext{
${ }^{1}$ Department of Pediatrics, University of Eastern Finland and Kuopio University Hospital, Kuopio, Finland. Correspondence: Henrikki Nordman (henrikki.nordman@kuh.fi)

Received 14 November 2016; accepted 6 February 2017; advance online publication 17 May 2017. doi:10.1038/pr.2017.44
} 


\section{Articles | Nordman et al.}

Table 1. Anthropometric and biochemical characteristics of the study groups

\begin{tabular}{|c|c|c|c|c|}
\hline & LGA & AGA & SGA & $P$ \\
\hline Total number (boys) & $49(25)$ & $56(29)$ & $23(13)$ & \\
\hline \multicolumn{5}{|l|}{ At birth } \\
\hline Gestational age (weeks) & $39.8(39.5-40.1)$ & $39.9(39.6-40.2)$ & $39.7(39.2-40.3)$ & 0.81 \\
\hline Weight (g) & 4722 (4631-4812) & 3561 (3484-3637) & $2476(2345-2607)$ & $<0.001$ \\
\hline Weight (SDS) & $2.63(2.46-2.79)$ & $-0.02(-0.16-0.12)$ & $-2.39(-2.53--2.25)$ & $<0.001$ \\
\hline Length $(\mathrm{cm})$ & $53.0(52.6-53.4)$ & $50.0(49.7-50.4)$ & $46.2(45.5-46.9)$ & $<0.001$ \\
\hline Length (SDS) & $1.58(1.40-1.76)$ & $-0.11(-0.24-0.03)$ & $-2.16(-2.43--1.88)$ & $<0.001$ \\
\hline \multicolumn{5}{|l|}{ At the age of 2 years } \\
\hline Weight (SDS) & $0.65(0.41-0.90)$ & $0.16(-0.14-0.45)$ & $-0.95(-1.37--0.53)$ & $<0.001^{\mathrm{a}}$ \\
\hline Height (SDS) & $0.40(0.17-0.63)$ & $-0.05(-0.34-0.24)$ & $-0.98(-1.33--0.63)$ & $<0.001^{\mathrm{a}}$ \\
\hline \multicolumn{5}{|l|}{ At examination } \\
\hline Age (years) & $6.89(6.62-7.16)$ & $7.09(6.86-7.33)$ & $6.65(6.22-7.07)$ & 0.13 \\
\hline Weight $(\mathrm{kg})$ & $27.6(26.2-29.0)$ & $27.5(25.7-29.4)$ & $21.8(19.8-23.7)$ & $<0.001$ \\
\hline Weight (SDS) & $0.68(0.38-0.97)$ & $0.39(0.11-0.67)$ & $-0.80(-1.26--0.33)$ & $<0.001$ \\
\hline Height $(\mathrm{cm})$ & $126.1(124.0-128.3)$ & $125.7(123.8-127.7)$ & $119.0(115.6-122.4)$ & $<0.001$ \\
\hline Height (SDS) & $0.54(0.30-0.78)$ & $0.20(-0.07-0.46)$ & $-0.64(-1.01--0.27)$ & $<0.001$ \\
\hline BMI (SDS) & $0.56(0.22-0.89)$ & $0.36(0.05-0.67)$ & $-0.65(-1.18--0.12)$ & $<0.001$ \\
\hline DHEAS $(\mu \mathrm{g} / \mathrm{dl})$ & $18.3(14.1-23.0)$ & $24.7(20.2-29.8)$ & $30.5(22.2-40.2)$ & $0.028^{\mathrm{b}}$ \\
\hline IGF-1 (ng/ml) & $196.3(179.1-214.8)$ & $198.6(182.4-216.3)$ & $190.1(165.2-218.8)$ & 0.87 \\
\hline Insulin $(\mu \mathrm{lU} / \mathrm{ml})$ & $4.47(3.92-5.09)$ & $4.79(4.24-5.40)$ & $4.84(3.94-5.93)$ & 0.70 \\
\hline
\end{tabular}

AGA, appropriate for gestational age; ANOVA, analysis of variance; BMI, body mass index; DHEAS, dehydroepiandrosterone sulfate; Cl, confidence interval; IGF-1; insulin-like growth factor 1; LGA, large for gestational age; SDS, standard deviation score; SGA, small for gestational age.

Data are presented as mean $(95 \% \mathrm{Cl}$ ), except DHEAS, IGF-1, and insulin (geometric mean (95\% CI)).

ANOVA between the three study groups, except DHEAS, IGF-1, and insulin (BMI SDS and age-adjusted ANCOVA).

The post hoc test (Sidak correction) $P<0.001$ between SGA and AGA/LGA groups; $P<0.05$ between LGA and AGA groups.

${ }^{b}$ The post hoc test (Sidak correction) $P=0.041$ between the LGA and SGA groups.

concentrations were determined using an ELISA kit (Mediagnost, Reutlingen, Germany).

A written informed consent was obtained from all the parents and from participating children aged $\geqslant 6$ years. The study protocol was approved by the Committee on Research Ethics of the Hospital District of Northern Savo.

\section{Statistical Analyses}

Data are presented as mean $(95 \% \mathrm{CI})$. Analyses were performed using SPSS statistical software (version 22; SPSS, IBM, Armonk, NY). A significance level of 0.05 was used in all analyses. Analysis of variance was used for comparisons between groups on anthropometric measures. Differences in serum DHEAS, IGF-1, and insulin concentrations between the three groups were analyzed by the BMI SDS and age-adjusted analysis of covariance and predictors of serum DHEAS levels (model 1: weight SDS at birth; model 2: the change in weight SDS from birth to the age of 2 years; both models: BMI SDS at examination, serum IGF-1, serum insulin, and age at examination) were explored by linear regression analysis. Skewed data were either logarithm- or square-root-transformed before parametric analyses. Obtained estimated means (BMI SDS and age-adjusted) were powertransformed to geometric means for presentation. Association of insulin or IGF-1 levels (tertiles) with the absence/presence of biochemical adrenarche (blood samples obtained at the same age;
DHEAS $<37 \mu \mathrm{g} / \mathrm{dl}(1.0 \mu \mathrm{mol} / \mathrm{l}) ;$ DHEAS $\geqslant 37 \mu \mathrm{g} / \mathrm{dl}(1.0 \mu \mathrm{mol} / \mathrm{l}))$ was analyzed using the $\chi^{2}$-test.

\section{RESULTS}

All girls had prepubertal breast stage (Tanner B1), all boys had prepubertal genital stage (Tanner G1), and no child had pubic hair. Nine girls (prevalence 14.8\%) and two boys (3.0\%) had some clinical sign of PA (six girls and two boys-adulttype body odor, two girls-oily hair, and one-comedones). Thirty-two children (25\%, 17 boys; 8 LGA, 18 AGA, and 6 SGA ( $P=0.17$, the $\chi^{2}$-test between the groups)) had DHEAS level $\geqslant 37 \mu \mathrm{g} / \mathrm{dl}(1.0 \mu \mathrm{mol} / \mathrm{l})$. Three girls and one boy $(3 \%)$ had both clinical signs of PA and DHEAS level $\geqslant 37 \mu \mathrm{g} / \mathrm{dl}$ $(1.0 \mu \mathrm{mol} / \mathrm{l})$.

Serum DHEAS levels did not differ between the sexes $(P=0.50)$. There was a significant difference in serum DHEAS levels between the three study groups (Table 1). The post hoc test (Sidak correction) showed significantly lower serum DHEAS levels in the LGA than in the SGA group (Table 1). A significant difference was also found in body mass index (BMI) standard deviation score (SDS) and 


\section{Birth size and serum DHEAS levels $\quad$ Articles}

Table 2. Determinants of serum DHEAS levels (linear regression analysis) in the whole study population at examination

\begin{tabular}{|c|c|c|c|c|c|}
\hline & Independent variables & Standardized $B$ & $P$ (variables) & $P$ (model) & $R^{2}$ \\
\hline \multirow[t]{3}{*}{ Model $1(n=125)$} & Weight SDS (at birth) & -0.27 & 0.002 & $<0.001$ & 0.27 \\
\hline & BMI SDS & 0.11 & 0.22 & & \\
\hline & Serum insulin & 0.03 & 0.73 & & \\
\hline \multirow[t]{3}{*}{ Model $2(n=123)$} & $\Delta$ Weight SDS $0-2$ years & 0.26 & 0.001 & $<0.001$ & 0.28 \\
\hline & Serum IGF-1 & 0.14 & 0.12 & & \\
\hline & Serum insulin & 0.03 & 0.78 & & \\
\hline
\end{tabular}

Adjusted for age at examination

age-adjusted serum DHEAS levels between the LGA and the rest of the study population $(P=0.015$; mean $(95 \%$ confidence interval (CI)), 18.4 (14.2-23.1); 26.2 (22.2-30.6), respectively). A trend toward lower DHEAS levels adjusted for BMI SDS and age was seen in children born LGA compared with those in children born AGA, but the difference was not statistically significant $(P=0.06$; Table 1). There were no significant differences in serum insulin-like growth factor 1 (IGF-1) and insulin concentrations between the study groups (Table 1).

Lower birth weight SDS (model 1) and higher weight gain during the first 2 years of life (model 2) predicted higher serum DHEAS concentrations in linear regression analyses (Table 2). The same trend was also seen when the children were arranged by their birth size or weight gain during the first 2 years of life and their weight SDS at examination (Figure 1). A significant association was found between biochemical adrenarche and IGF-1 levels $(P=0.007)$, but not between biochemical adrenarche and insulin levels $(P=0.57$; Table 3).

\section{DISCUSSION}

As far as we know, there are no previously published reports on DHEAS levels or early adrenarche in LGA-born children. In this study, the prepubertal children born LGA had lower serum DHEAS levels compared with children born SGA or children born SGA and AGA combined. Low birth weight SDS and early weight SDS change (0-2 years) were associated with DHEAS levels, and the prevalence of biochemical adrenarche was highest among the children with the highest IGF-1 levels. There was no difference in the DHEAS levels between the sexes.

Previous studies have shown an association between low birth size and increased serum DHEAS levels in later childhood $(1,2,26)$. In addition, the Avon Longitudinal Study of Parents and Children study with a large population-based sample of children showed that the negative relationship between birth weight and later prepubertal serum DHEAS levels is continuous throughout the normal birth weight range (20). Our study shows that the trend toward low prepubertal DHEAS levels extends to the LGA-born children. Consequently, children born LGA are not prone to develop PA that has been linked to a later metabolic risk (6-9).
Table 3. Association of biochemical adrenarche with IGF-1 and insulin levels at examination

\begin{tabular}{|c|c|c|c|c|c|c|}
\hline \multirow[t]{2}{*}{ Biochemical adrenarche } & \multicolumn{2}{|c|}{1} & \multicolumn{2}{|c|}{2} & \multicolumn{2}{|c|}{3} \\
\hline & $n$ & $\%$ & $n$ & $\%$ & $n$ & $\%$ \\
\hline \multicolumn{7}{|l|}{ IGF-1 tertiles } \\
\hline Yes & 7 & 21.9 & 7 & 21.9 & 18 & 56.3 \\
\hline No & 34 & 36.6 & 35 & 37.6 & 24 & 25.8 \\
\hline
\end{tabular}

Insulin tertiles

$\begin{array}{lllllll}\text { Yes } & 9 & 28.1 & 10 & 31.3 & 13 & 40.6\end{array}$

\begin{tabular}{crrrrrr} 
No & 33 & 34.7 & 33 & 34.7 & 29 & 30.5 \\
\hline The $x^{2}$-test between IGF-1 tertiles, $P=0.007$, and insulin tertiles, $P=0.57$.
\end{tabular} Biochemical adrenarche: serum DHEAS $\geqslant 37 \mu \mathrm{g} / \mathrm{dl}(\geqslant 1 \mu \mathrm{mol} / \mathrm{l})$.

Adrenal androgen levels do not have the U-shaped association with birth size as some other metabolic risks and outcomes may have $(10,11)$. As no follow-up studies exist, the later significance of lower DHEAS levels in children born LGA remains unknown.

Metabolic programming during fetal (e.g., intrauterine growth restriction) and early postnatal development, as well as increased IGF-1 and insulin levels in SGA-born children with catch-up growth, has been linked to increased prepubertal adrenal androgen secretion (20). These factors could at least partly explain the negative association between birth weight and later serum DHEAS levels, as the majority of the SGA- and LGA-born children experience catch-up and catch-down growth, respectively. This might indicate a greater impact of the early childhood growth pattern than the birth size itself on later adrenal androgen production and its consequences.

Our data did not show difference in the DHEAS levels between the sexes. In a previous study, girls had higher DHEAS than boys had, but, in that study, DHEAS was not adjusted for BMI or age (27). When these factors are taken into account, no difference in serum DHEAS between the sexes is expected (24).

In our study, children born SGA and AGA did not have a significant difference in their DHEAS levels, but birth weight was negatively associated with prepubertal DHEAS levels in the whole study population, supporting the previously described association $(1,2)$. One reason for the relatively high 


\section{Articles | Nordman et $a$ al.}
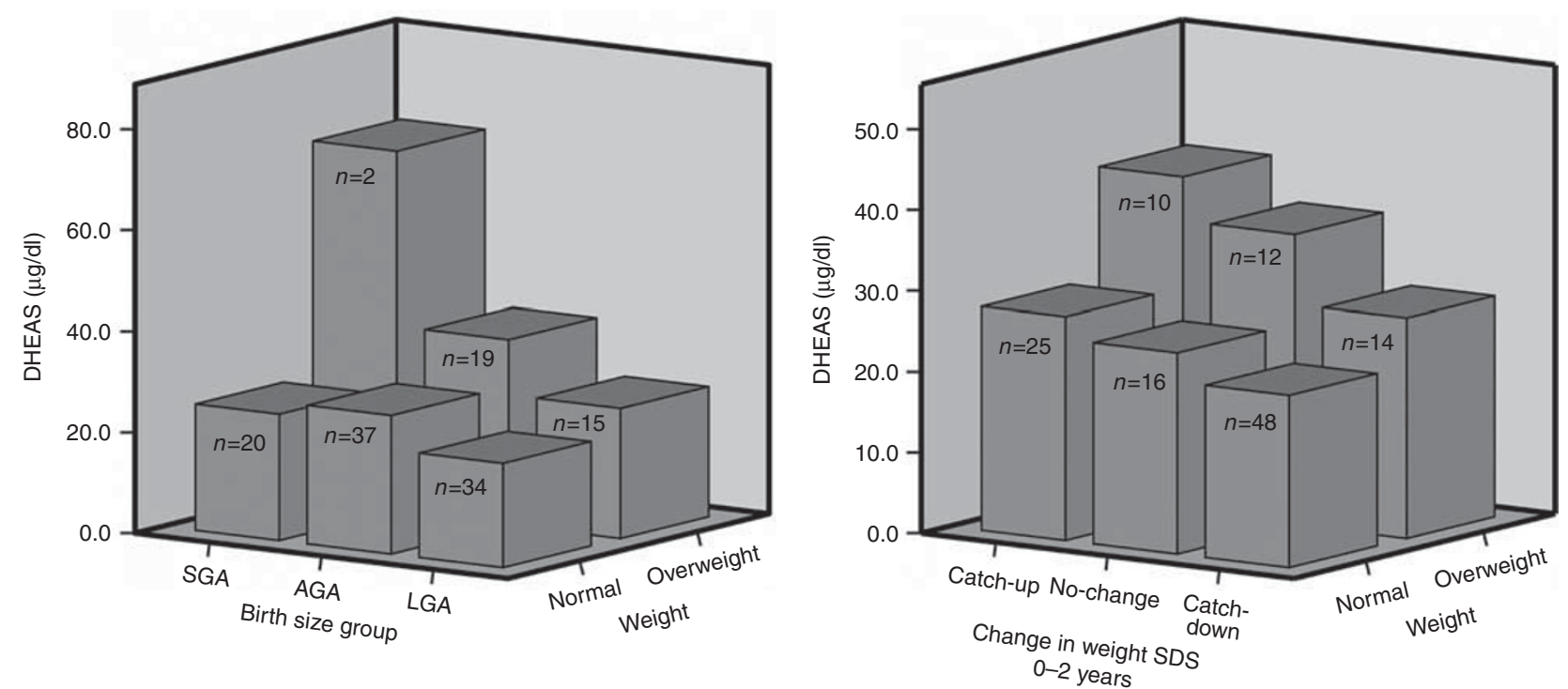

Figure 1. Dehydroepiandrosterone sulfate (DHEAS) levels (mean) at prepuberty, by birth size/early childhood weight development, and current weight. Normal and overweight defined as body mass index (BMI) standard deviation score (SDS) corresponding to adult BMI $<25$ and $\geqslant 25$, respectively (22).

DHEAS levels in our children born AGA may be their BMI SDS being above the population mean (21) at the examination. Early catch-up growth and weight in childhood seem to have a strong impact on DHEAS levels in prepuberty $(20,28)$, which was also noticeable in our study.

No child in this study had pubic or axillary hair, which is permissible as the appearance of the clinical signs of PA is related to the level of androgen effect (ascending order: adulttype body odor, oily hair, comedones, and pubic or axillary hair) (25), although variation may occur because of ethnicity. The prevalence of children with some clinical sign of PA and of girls with both clinical signs of PA and DHEAS level $\geqslant 37 \mu \mathrm{g} / \mathrm{dl}$ was lower in our study than in the previous Finnish study of larger population (24). Participants being younger in our study could explain the difference. In addition, the proportion of children with biochemical adrenarche was higher in our study compared with that in the data by Mäntyselkä et al (24). Increased body weight is associated with PA $(9,17)$, and the children belonging to LGA and AGA groups were somewhat heavier in our study than the children in the previous study (24). Our findings are thus well coherent with the previously published Finnish data, although some differences could originate from the relatively small sample size in the current study.

Increased IGF-1 concentrations were previously demonstrated in prepubertal girls $(19,29)$ and boys (27) with PA compared with those in age-matched controls. IGF-1 stimulates steroidogenesis in adrenocortical cells, and its serum concentrations increase with overweight and hyperinsulinism (reviewed in (refs $3,4)$ ). In our study, linear regression analysis showed a trend between serum IGF-1 and DHEAS levels, and the highest prevalence of biochemical adrenarche was detected in children with the highest IGF-1 serum levels. Hyperinsulinism has also been suggested to contribute to PA (4). However, our study showed no association of insulin with DHEAS. In our previous study, prepubertal girls with PA had slightly higher oral glucose tolerance test-stimulated serum insulin concentrations than control girls had, but basal insulin concentrations were similar (7).

The strengths of this study include the detailed physical examination including the clinical signs of adrenarche and the pubertal stage. In addition, the study participants were enrolled strictly according to the birth size, and the AGA group was selected to represent the children close to the mean birth weight and length. Furthermore, to reduce the influence of confounding factors, we adjusted our analyses for BMI SDS and age. The main limitation of this study is the relatively small sample size, affecting the statistical power of the study.

In conclusion, being born LGA was associated with lower prepubertal DHEAS levels, whereas small birth size and early catch-up growth predicted increased DHEAS levels. This suggests that genetic or early epigenetic factors have an impact on adrenal androgen secretion and adrenarche. IGF-1 may be a mediator in this process.

\section{Disclosure: The authors declare no conflict of interest.}

\section{REFERENCES}

1. Tenhola S, Martikainen A, Rahiala E, Parviainen M, Halonen P, Voutilainen R. Increased adrenocortical and adrenomedullary hormonal activity in 12-year-old children born small for gestational age. J Pediatr 2002;141:477-82.

2. Ibáñez L, Lopez-Bermejo A, Díaz M, Suárez L, de Zegher F. Low-birth weight children develop lower sex hormone binding globulin and higher dehydroepiandrosterone sulfate levels and aggravate their visceral adiposity and hypoadiponectinemia between six and eight years of age. J Clin Endocrinol Metab 2009;94:3696-9.

3. Idkowiak J, Lavery GG, Dhir V, et al. Premature adrenarche: novel lessons from early onset androgen excess. Eur J Endocrinol 2011;165:189-207. 
4. Voutilainen R, Jääskeläinen J. Premature adrenarche: etiology, clinical findings, and consequences. J Steroid Biochem Mol Biol 2015;145:226-36.

5. Utriainen $\mathrm{P}$, Laakso $\mathrm{S}$, Liimatta $\mathrm{J}$, Jääskeläinen $\mathrm{J}$, Voutilainen $\mathrm{R}$. Premature adrenarche-a common condition with variable presentation. Horm Res Paediatr 2015;83:221-31.

6. Oppenheimer E, Linder B, DiMartino-Nardi J. Decreased insulin sensitivity in prepubertal girls with premature adrenarche and acanthosis nigricans. J Clin Endocrinol Metab 1995;80:614-8.

7. Utriainen P, Jääskeläinen J, Romppanen J, Voutilainen R. Childhood metabolic syndrome and its components in premature adrenarche. J Clin Endocrinol Metab 2007;92:4282-5.

8. Ibáñez L, Potau N, Chacon P, Pascual C, Carrascosa A. Hyperinsulinaemia, dyslipaemia and cardiovascular risk in girls with a history of premature pubarche. Diabetologia 1998;41:1057-63.

9. Utriainen P, Jääskeläinen J, Saarinen A, Vanninen E, Mäkitie O, Voutilainen R. Body composition and bone mineral density in children with premature adrenarche and the association of LRP5 gene polymorphisms with bone mineral density. J Clin Endocrinol Metab 2009;94:4144-51.

10. Harder T, Rodekamp E, Schellong K, Dudenhausen JW, Plagemann A. Birth weight and subsequent risk of type 2 diabetes: a meta-analysis. Am J Epidemiol 2007;165:849-57.

11. Whincup PH, Kaye SJ, Owen CG, et al. Birth weight and risk of type 2 diabetes: a systematic review. JAMA 2008;300:2886-97.

12. Saenger P, Czernichow P, Hughes I, Reiter EO. Small for gestational age: short stature and beyond. Endocr Rev 2007;28:219-51.

13. Evagelidou EN, Giapros VI, Challa AS, et al. Prothrombotic state, cardiovascular, and metabolic syndrome risk factors in prepubertal children born large for gestational age. Diabetes Care 2010;33:2468-70.

14. Bowers K, Liu G, Wang P, et al. Birth weight, postnatal weight change, and risk for high blood pressure among Chinese children. Pediatrics 2011;127:e1272-9.

15. Taal HR, Vd Heijden AJ, Steegers EA, Hofman A, Jaddoe VW. Small and large size for gestational age at birth, infant growth, and childhood overweight. Obesity 2013;21:1261-8.

16. Johnsson IW, Haglund B, Ahlsson F, Gustafsson J. A high birth weight is associated with increased risk of type 2 diabetes and obesity. Pediatr Obes 2015;10:77-83.
17. Neville KA, Walker JL. Precocious pubarche is associated with SGA, prematurity, weight gain, and obesity. Arch Dis Child 2005;90:258-61.

18. Pere A, Perheentupa J, Peter M, Voutilainen R. Follow up of growth and steroids in premature adrenarche. Eur J Pediatr 1995;154:346-52.

19. Utriainen P, Voutilainen R, Jäskeläinen J. Girls with premature adrenarche have accelerated early childhood growth. J Pediatr 2009;154: $882-7$.

20. Ong KK, Potau N, Petry CJ, et al. Opposing influences of prenatal and postnatal weight gain on adrenarche in normal boys and girls. J Clin Endocrinol Metab 2004;89:2647-51.

21. Nordman H, Voutilainen R, Laitinen T, et al. Growth and cardiovascular risk factors in prepubertal children born large or small for gestational age. Horm Res Paediatr 2016;85:11-7.

22. Saari A, Sankilampi U, Hannila ML, Kiviniemi V, Kesseli K, Dunkel L. New Finnish growth references for children and adolescents aged 0 to 20 years: length/height-for-age, weight-for-length/height, and body mass index-for-age. Ann Med 2011;43:235-48.

23. Monteiro PO, Victora CG. Rapid growth in infancy and childhood and obesity in later life - a systematic review. Obes Rev 2005;6:143-54.

24. Mäntyselkä A, Jääskeläinen J, Lindi V, et al. The presentation of adrenarche is sexually dimorphic and modified by body adiposity. J Clin Endocrinol Metab 2014;99:3889-94.

25. Utriainen P, Voutilainen R, Jääskeläinen J. Continuum of phenotypes and sympathoadrenal function in premature adrenarche. Eur $\mathrm{J}$ Endocrinol 2009;160:657-65.

26. Schulte S, Wölfle J, Schreiner F, et al. Birthweight differences in monozygotic twins influence pubertal maturation and near final height. J Pediatr 2016;170:288-94. e1-2.

27. Denburg MR, Silfen ME, Manibo AM, et al. Insulin sensitivity and the insulin-like growth factor system in prepubertal boys with premature adrenarche. J Clin Endocrinol Metab 2002;87:5604-9.

28. Corvalán C, Uauy R, Mericq V. Obesity is positively associated with dehydroepiandrosterone sulfate concentrations at $7 \mathrm{y}$ in Chilean children of normal birth weight. Am J Clin Nutr 2013;97:318-25.

29. Silfen ME, Manibo AM, Ferin M, McMahon DJ, Levine LS, Oberfield SE. Elevated free IGF-I levels in prepubertal Hispanic girls with premature adrenarche: relationship with hyperandrogenism and insulin sensitivity. J Clin Endocrinol Metab 2002;87:398-403. 